\title{
Fractura con hundimiento de cráneo intrauterina espontánea: reporte de dos casos
} Spontaneous intrauterine depressed skull fracture: report of two cases

\author{
Sofía C. Geffner ${ }^{a} \mathbb{D}$, Luisa M. Baldini ${ }^{a}$ (D) Cecilia Péreza ${ }^{a}$
}

\begin{abstract}
RESUMEN
Las fracturas con hundimiento de cráneo intrauterinas representan una entidad poco frecuente, generalmente secundaria a traumatismos (previos o durante el nacimiento) o de etiología desconocida. Suelen requerir evaluación y seguimiento por el servicio de Neurocirugía Pediátrica. A la fecha, es controversial la necesidad de tratamiento quirúrgico y el momento oportuno para concretarlo.

Se presentan dos casos clínicos de pacientes de término, nacidas por cesárea, con diagnóstico posnatal inmediato dehundimiento de cráneo de tipo ping-pong no traumático.

Ambas pacientes presentaron examen neurológico normal. Se confirmó el diagnóstico a través de radiografía y tomografía de cráneo, sin observarse lesiones asociadas. Fueron valoradas por el servicio de Neurocirugía, que indicó corrección quirúrgica de la lesión en ambos casos, con buena evolución posterior.

Palabras clave: fractura craneal deprimida, anomalías congénitas, fracturas espontáneas.
\end{abstract}

\begin{abstract}
Spontaneous intrauterine depressed skull fractures are a rare entity. They can appear secondarily to head trauma (before or during birth) or due to unknown etiology. They usually require a complete evaluation from pediatric neurosurgery specialists. Their optimal management, including timely surgical treatment remains controversial.

We describe two cases delivered by cesarean section, with postnatal diagnosis of spontaneous intrauterine depressed skull fracture. Both had a normal neurological exam. A skull radiography and head CT were performed, and no associated lesions were found. Both cases required surgical correction, with positive results.

Key words: skull fracture depressed, congenital abnormalities, fractures, spontaneous.
\end{abstract}

http: / / dx.doi.org/10.5546/ aap.2022.e85

Cómo citar: Geffner SC, Baldini LM, Pérez C. Fractura con hundimiento de cráneo intrauterina espontánea: reporte de dos casos. Arch Argent Pediatr 2022;120(2):e85-e88.

a. Servicio de Neonatología, Hospital Italiano de Buenos Aires, Ciudad Autónoma de Buenos Aires, Argentina.

\section{Correspondencia:}

Sofía C. Geffner: sofia.geffner@hospitalitaliano.org.ar

Financiamiento: Ninguno.

Conflicto de intereses: Ninguno que declarar.

Recibido: 15-4-2021

Aceptado: 12-8-2021

\section{INTRODUCCIÓN}

Las fracturas intrauterinas con hundimiento de cráneo o de tipo ping-pong son depresiones congénitas craneales, cuya incidencia es incierta. Se pueden clasificar como traumáticas o no traumáticas. Las primeras son las más frecuentes y pueden estar asociadas a lesiones en el parénquima encefálico. ${ }^{1-3} \mathrm{El}$ segundo grupo, las formas espontáneas o idiopáticas, son menos habituales y su etiología no suele ser evidente. ${ }^{2}$

Constituyen una entidad poco frecuente y la conducta por seguir es, a la fecha, controversial. Ante este diagnóstico se sugiere realizar una valoración por parte de Neurocirugía para definir la necesidad de corrección quirúrgica. ${ }^{1,2}$

Se reportan aquí los casos de dos pacientes con diagnóstico de fractura con hundimiento de cráneo espontánea, sin lesiones asociadas en el parénquima encefálico. Ambas fueron valoradas por Neurocirugía y corregidas de forma quirúrgica, con buena evolución posterior.

\section{CASO CLÍNICO 1}

Recién nacida de término (39,2 semanas de edad gestacional), peso adecuado para la edad gestacional (2995 gramos), embarazo controlado, nacida por cesárea electiva sin complicaciones y sin requerimiento de instrumentación para la extracción. Al momento del examen físico, se palpó depresión parietal derecha de $3 \mathrm{~cm}$ de diámetro, de fondo duro, no parecía dolorosa a la palpación. Perímetro cefálico de $34 \mathrm{~cm}$ (percentil 67 para edad gestacional). La madre negó traumatismo abdominal previo al nacimiento. Se constató examen neurológico normal: pupilas isocóricas y reactivas, movimientos oculares conservados, facies simétrica, movimientos en los 4 miembros conservados y succión vigorosa al pecho materno.

Se solicitó evaluación al servicio de Neurocirugía, que decidió realizar tomografía computada de cerebro con reconstrucción 3D (Figuras 1A y 1B), que informó hundimiento de cráneo focal parietal derecho de $3 \mathrm{~cm}$ de diámetro aproximadamente con probable trazo de fractura lineal en la convexidad. No se observaron otras 
lesiones intracraneales: se constataron ventrículos de forma, tamaño y posición normales, sin colecciones ni hemorragias subyacentes. Hipótesis diagnóstica: fractura de tipo ping-pong.

Debido a la magnitud de la lesión (asociada a menor tasa de recuperación espontánea), se indicó corrección quirúrgica al sexto día de vida. Técnica quirúrgica: incisión parietal derecha adyacente al eje mayor de la fractura, trepanación de $7 \mathrm{~mm}$ y elevación subcraneal extradural del hundimiento. La paciente toleró adecuadamente el procedimiento.

Como parte del plan de estudio, se solicitó evaluación al servicio de Genética. Se realizó radiografía de huesos largos y de columna, sin hallazgos patológicos. Por buena evolución clínica, se otorgó el egreso hospitalario a las 48 horas de la intervención quirúrgica.

A los 4 meses de vida, la paciente concurrió a control. Se observó buena evolución clínica, buen progreso pondoestatural y neurodesarrollo adecuado. Se constató plagiocefalia occipital izquierda, por lo que se indicaron cambios posturales. Continúa con controles ambulatorios en los servicios de Pediatría y Neurocirugía.

\section{CASO CLÍNICO 2}

Recién nacida de término $(40,4$ semanas de edad gestacional), peso adecuado para la edad gestacional (3425 gramos), producto de un embarazo controlado, sin antecedentes de relevancia. Nacida por cesárea con presentación cefálica, por falta de progresión y descenso, luego de 17 horas de trabajo de parto.

Al momento del nacimiento, se palpó depresión parietal izquierda de $2 \mathrm{~cm}$ de diámetro aproximadamente. Se constató examen físico neurológico dentro de parámetros normales. Perímetro cefálico: $36 \mathrm{~cm}$ (percentil 96 para edad gestacional).

Fue evaluada por el servicio de Neurocirugía, que solicitó radiografía (Figura 2) y tomografía computada de cráneo con reconstrucción 3D (Figura 3A). Se observó hundimiento parietal izquierdo, sin trazos de fractura evidentes, sin alteraciones en el parénquima encefálico (Figuras 3B y 3C). Se decidió intervención quirúrgica para la corrección de la lesión a los 7 días de vida. Técnica quirúrgica mediante craniectomía mínima parietal con levantamiento del hundimiento craneal. La paciente toleró adecuadamente la intervención y se otorgó el egreso hospitalario a las 48 horas del procedimiento. Al mes de vida, la paciente presenta buena evolución clínica y neurodesarrollo acorde a su edad.

\section{DISCUSIÓN}

Las fracturas intrauterinas con hundimiento o de tipo ping-pong son depresiones congénitas craneales. La incidencia es incierta; se han reportado frecuencias de 1 a 2,5/10 000 nacidos

Figura 1. Tomografía axial computada de cráneo de la paciente del caso clínico 1. A: reconstrucción en 3D en la que se observa hundimiento parietal derecho, con probable trazo fracturario en su concavidad. B: corte transversal que muestra hundimiento parietal derecho.
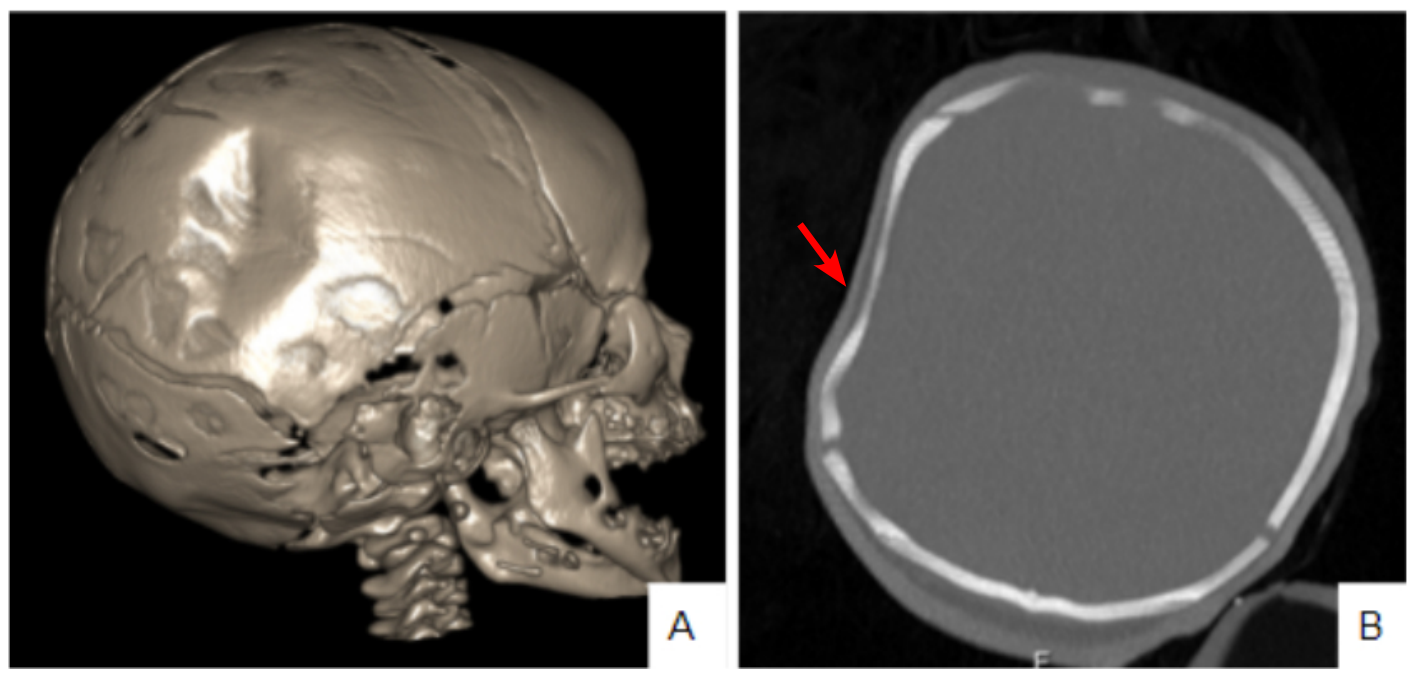
vivos, ${ }^{1} 1 / 4000$ nacidos vivos ${ }^{2}$ y hasta 3,7 / 100000 nacidos vivos. ${ }^{3}$

Existen dos tipos: traumáticas o no traumáticas. Las primeras son las más frecuentes y se deben a trauma abdominal previo o lesión durante el nacimiento. Se incluyen en esta categoría las lesiones por partos instrumentados o cesáreas. En cuanto al segundo grupo, reciben el nombre de fracturas con hundimiento intrauterinas espontáneas, son menos frecuentes y tienen etiologías menos evidentes: presión por parte del promontorio del hueso sacro, exostosis materna, fibroma o mioma uterino, o traumatismo en el pasaje a través del canal de parto. ${ }^{3}$ En ocasiones, ha mostrado asociación con oligohidramnios. ${ }^{1}$ La mineralización reducida y la elevada maleabilidad ósea pueden ser, en parte, responsables del moldeamiento secundario a la compresión externa. No siempre se reporta un trazo fracturario con pérdida de continuidad del hueso; en ocasiones se describe solo un hundimiento óseo. ${ }^{4}$

En pacientes que presentan este diagnóstico, se debe evaluar la posibilidad de estar frente a una fractura patológica secundaria a displasias óseas o enfermedades del colágeno.

La localización de estas fracturas es más frecuente en el hueso frontal $(65 \%)$, seguido por el hueso parietal $(28 \%) .^{5}$

Frente a pacientes con fractura con hundimiento de cráneo intrauterina, se recomienda realizar radiografía y tomografía computada de cráneo, con el objetivo de evaluar la presencia de lesiones asociadas, como hematoma epidural o subdural y daño parenquimatoso, y la presencia de fragmentos óseos. ${ }^{3}$

En cuanto al pronóstico y a la evolución, las formas espontáneas suelen ser benignas y no se asocian a daño neurológico. ${ }^{1,3}$ Sin embargo, las formas iatrogénicas o traumáticas pueden

FIGURA 2. Radiografía de cráneo frente de la paciente del caso clínico 2

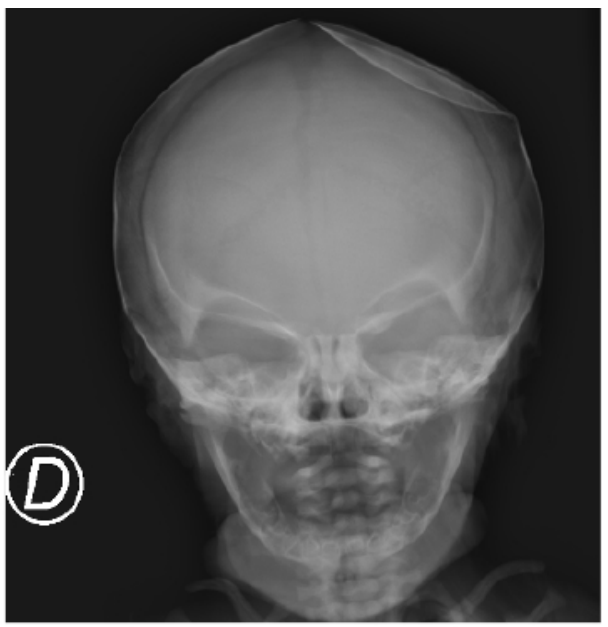

FIgURA 3. Tomografía axial computada de cráneo y cerebro de la paciente presentada en el caso clínico 2. A: reconstrucción en 3D que muestra hundimiento parietal izquierdo. B y C: corte transversal y coronal que muestra la lesión.
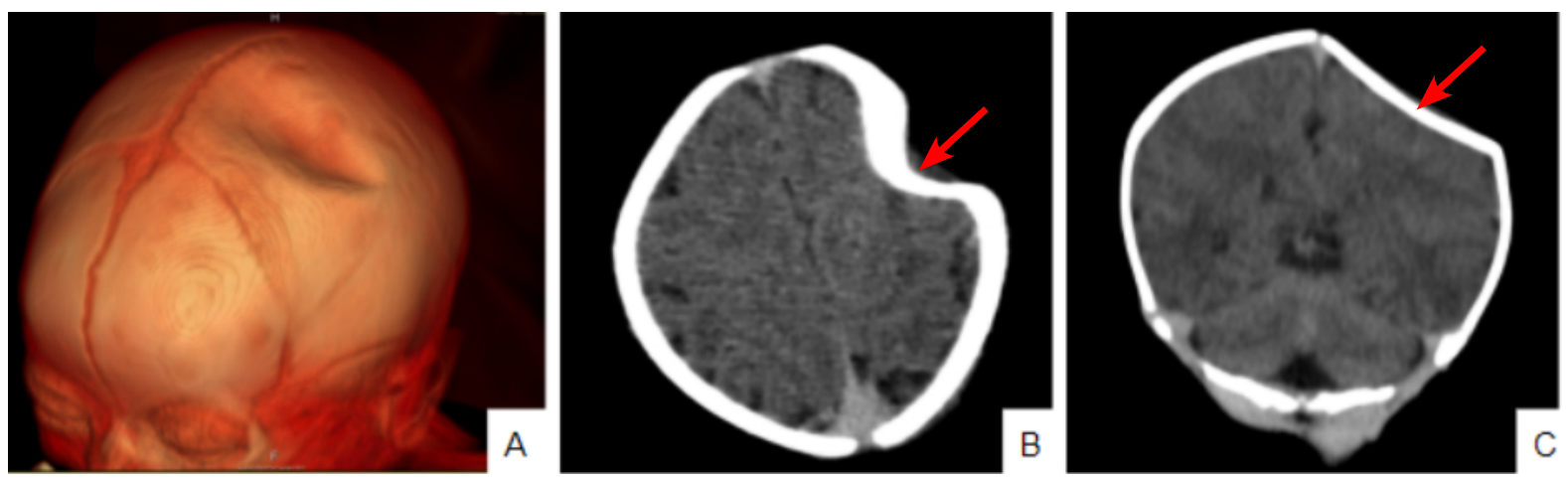
relacionarse con lesiones en el parénquima encefálico, como compresión o hemorragia cerebral con atrofia cortical y gliosis. ${ }^{1}$ En la revisión realizada por Dupuis y cols., ${ }^{3}$ se reporta que hasta un $30 \%$ de los pacientes con fracturas iatrogénicas presentan algún tipo de daño encefálico, por lo que se recomienda la realización de tomografía de cráneo, mientras que en las formas espontáneas no se vio asociación. Las estructuras subyacentes al cráneo (duramadre y grandes vasos) no suelen ser maleables y esta podría ser la causa de las lesiones producidas en el parénquima encefálico. En ocasiones, el daño neurológico puede provocar secuelas a largo plazo; Dupuis y cols., ${ }^{3}$ reportan un $4 \%$ de secuelas en los casos de fracturas de cráneo iatrogénicas. Los autores describen un caso de tetraparesia espástica y otro de hemiparesia. Otras complicaciones asociadas están representadas por alteraciones estéticas producidas a expensas de dichas fracturas, observadas con mayor frecuencia en las formas iatrogénicas. ${ }^{3}$

Frente al diagnóstico de las fracturas con hundimiento de cráneo, la recomendación actual es realizar una evaluación clínica completa y una evaluación por parte de un neurocirujano a fin de definir la necesidad de resolución quirúrgica. Esta decisión no resulta sencilla. Algunos autores recomiendan seguimiento clínico; de hecho, se han reportado casos de resolución espontánea de la lesión..$^{5-7}$

Una conducta alternativa favorece la corrección quirúrgica, ${ }^{1,2,8}$ cuyas indicaciones son lesiones asociadas a la fractura (presencia de hemorragia encefálica, contusión cerebral o fragmentos óseos), signos de foco neurológico asociado a la fractura, hipertensión endocraneal, gran defecto estético o lesiones de gran tamaño (más de $5 \mathrm{~mm}$ de profundidad y/o más de $2 \mathrm{~cm}$ de largo) por el riesgo de compresión cerebral y aparición de convulsiones. ${ }^{9}$ En ausencia de estas indicaciones, hay autores que recomiendan esperar desde días hasta meses luego del diagnóstico a fin de realizar la corrección quirúrgica. ${ }^{2,3}$

En resumen, las fracturas con hundimiento de cráneo intrauterinas son una entidad de diagnóstico extremadamente rara. Pueden ser secundarias a un traumatismo o, con menor frecuente, ser espontáneas. Requieren de evaluación conjunta por parte de un pediatra y un neurocirujano, a fin de decidir la conducta por seguir: control clínico o tratamiento quirúrgico. Los pacientes suelen presentar buena evolución posterior, particularmente en las formas no traumáticas de estas fracturas.

\section{Agradecimiento:}

Al Dr. Nicolás Montivero, neurocirujano pediátrico en el Hospital Italiano de Buenos Aires.

\section{REFERENCIAS}

1. Veeravagu A, Azad T, Jiang B, Edwards M. Spontaneous Intrauterine Depressed Skull Fractures: Report of 2 Cases Requiring Neurosurgical Intervention and Literature Review. World Neurosurg. 2018; 110:256-62.

2. Aliabadi H, Miller J, Radnakrishnan S, Mahta AI, et al. Spontaneous Intrauterine "Ping-Pong" Fracture: Review and Case Illustration. Neuropediatrics. 2009; 40(2):73-5.

3. Dupuis O, Silveira R, Dupont C, Mottolese C, et al. Comparison of "instrument-associated" and "spontaneous" obstetric depressed skull fractures in a cohort of 68 neonates. Am J Obstet Gynecol. 2005; 192(1):165-70.

4. Axton JH, Levy LF. Congenital Moulding Depressions of the Skull. Br Med J. 1965; 1(5451):1644-7.

5. Basaldella L, Marton E, Bekelis K, Longatti P.Spontaneous resolution of atraumatic intrauterine ping-pong fractures in newborns delivered by cesarean section. J Child Neurol. 2011; 26(11):1449-51.

6. Preston D, Jackson S, Gandhi S. Non-traumatic depressed skull fracture in a neonate or 'ping pong' fracture. BMJ Case Rep. 2015; 2015:bcr2014207077.

7. Ilhan O, Bor M, Yukkaldiran P. Spontaneous resolution of a 'ping-pong' fracture at birth. BMJ Case Rep. 2018; 2018:bcr2018226264.

8. Moscote-Salazar LR, Bosco González J, Joaquim AF, Pacheco-Hernández A. Neurosurgical Management of Spontaneous Intrauterine Depressed Skull Fracture. World Neurosurg. 2018; 111:421.

9. Hung KL, Liao HT, Huang JS. Rational management of simple depressed skull fractures in infants. J Neurosurg. 2005; 103(1 Suppl):69-72. 